\title{
Réponses du zooplancton lacustre aux feux et aux coupes de forêt dans l'écozone boréale du Québec : étude préliminaire ${ }^{3}$
}

\author{
B. Pinel-Alloul ${ }^{1}$ \\ A. Patoine ${ }^{1}$ \\ R. Carignan ${ }^{1}$ \\ E. Prepas ${ }^{2}$
}

Mots-clés : forêt boréale, Québec, lacs, zooplancton, feux, coupes, impacts écologiques.

Les changements du zooplancton lacustre à la suite des feux et des coupes à blanc dans la forêt boréale ont été évalués en 1996 en comparant trois groupes de lacs du Québec méridional : 20 lacs de référence aux bassins versants non perturbés, 9 lacs ayant subi en 1995 des feux de forêt sur la majorité de leur bassin versant et 9 lacs ayant eu en 1995 une coupe à blanc sur une partie de leur bassin versant. Les communautés zooplanctoniques ont été caractérisées par différentes variables : 1) la composition, la richesse spécifique et la densité du zooplancton, 2) la masse organique du limnoplancton (seston $>53$ um) et 3) le biovolume du méso- et macrozooplancton $(>250 \mu \mathrm{m})$. La richesse spécifique et les assemblages d'espèces en juillet 1996 ne différaient pas entre les groupes de lacs ; la variation inter-réplicats dans les assemblages d'espèces de chaque lac était très faible en comparaison de la variation inter-lacs. Par contre, la densité du zooplancton et la masse organique du limnoplancton étaient en moyenne significativement plus élevées dans les lacs de feux que dans les lacs de coupes et les lacs de référence. Le biovolume du méso et macrozooplancton suivait les mêmes tendances. L'effet des traitements (groupes de lacs : référence, feu, coupe) expliquait moins de $15 \%$ de la variance totale de la masse organique du limnoplancton et du biovolume du méso et macrozooplancton tandis que les différences inter-lacs expliquaient la majorité $(>70 \%)$ de la variance totale dans ces indicateurs. Les $15 \%$ de variance résiduelle étaient attribuables à la variation inter-réplicats dans chaque lac. Notre étude préliminaire indique que les feux de forêts provoquent une poussée trophique du zooplancton et du limnoplancton mais n'ont pas d'effet sur la biodiversité et les assemblages d'espèces de zooplancton. Par contre, les coupes de forêt n'accroissent pas la biomasse du zooplancton en dépit d'une légère augmentation des nutriments, car les apports plus importants en carbone organique dissous après la coupe limitent la production biologique. Les changements limnologiques d'un lac à l'autre ont beaucoup plus d'importance que les feux et les coupes de forêts dans le contrôle environnemental du zooplancton des lacs de la forêt boréale.

Responses of lake zooplankton to natural fire and forest harvesting in the boreal ecozone in Quebec : preliminary study

Keywords : boreal forest, Quebec, zooplankton, natural fire, harvesting, ecological impacts.

Changes in lake zooplankton following forest fire and clear-cut harvesting in the boreal forest were investigated in 1996 by comparing three groups of lakes in southern Quebec : 20 reference lakes with unperturbed watersheds, 9 lakes impacted in 1995 by forest fires over most of their watersheds and 9 lakes impacted in 1995 by forest harvesting over a part of their watersheds. Zooplankton community has been characterized by different variables : 1) species richness, density and composition, 2) organic biomass of limnoplankton (seston $>53 \mu \mathrm{m}$ ) and 3) meso- and macrozooplankton biovolume $(>250 \mu \mathrm{m})$. Species richness and assemblages in July 1996 did not vary among groups of lakes ; inter-replicate variation in species assemblages within a lake was low compared to inter-lake variation. On the other hand, zooplankton density and total limnoplankton biomass were in average significantly higher in the lakes impacted by fire than in the reference lakes and lakes impacted by forest harvesting. Meso- and macrozooplankton biovolume followed a similar trend. The effect of treatments (groups of lakes: reference, fire, harvesting) explain less than $15 \%$ of total variance in limnoplankton and meso- and macrozooplankton whereas most of the variation ( $>70 \%)$ is related to changes among lake environments. The $15 \%$ of the residual variance was due to inter-replicate variation within a la-

1. Groupe de Recherche Interuniversitaire en Limnologie et en Environnement Aquatique (GRIL) et Centre d'Excellence en Gestion Durable de la Forêt, Département de sciences biologiques, Université de Montréal, C.P. 6128, Succ. Centre ville, Montréal, Qué. H3C 3J7, Canada.

2. Centre of Excellence for Sustainable Forest Management, Department of Biological Sciences, G-208 Biological Sciences Building, University of Alberta, Edmonton, Alberta,TG9 2E9, Canada.

3. Communication présentée à la 5 ème Conférence Internationale des Limnologues d'Expression Française (CLLEF-5), Namur- Belgique : 7-1 1 juitlet 1997. 
ke. Our preliminary study indicates that forest fires are related to a trophic upsurge of limnoplankton and meso- and macrozooplankton but have little influence on zooplankton biodiversity and species composition. In contrast, forest harvesting does not increase zooplankton variables, despite a slight increase in nutrients, because higher inputs of organic carbon after harvesting limits the biological production. Variations in limnological characteristics among lakes are more important for the environmental control of zooplankton community of boreal lakes than perturbations due to forest fire or harvesting.

\section{Introduction}

Le Canada renferme environ $10 \%$ de toutes les forêts du globe et $40 \%$ de la forêt boréale (Forestry Canada 1992). Formant la plus grande écozone du pays ( $35 \%$ du territoire canadien), la forêt boréale soutient la majorité des activités de l'industrie forestière qui compte parmi les plus importantes et contribue pour $3,2 \%$ au PNB et $15 \%$ aux exportations canadiennes (Statistics Canada 1994). Les perturbations majeures qui touchent les écosystèmes de la forêt boréale sont les feux et les coupes à blanc et, dans une moindre mesure, les épidémies d'insectes et les chablis (Johnson 1992, Hansson 1992). Par exemple, 988000 hectares de forêt ont été coupés chaque année au Canada de 1985 à 1990, dont $91 \%$ étaient des coupes à blanc (Forestry Canada 1992). Chaque année, on enregistre environ 9000 feux de forêts au Canada, ce qui représente 2,8 millions d'hectares brûlés, en grande majorité dans la forêt boréale (Canadian Geographic 1996).

Au Québec, on compte près d'un million de lacs dans la forêt boréale et ces milieux constituent des ressources importantes pour les habitats fauniques et la pêche sportive (Mc Nicol et al. 1987, in Papineau 1996). Toutefois, les impacts écologiques des feux et des coupes sur les lacs de la forêt boréale sont encore très peu connus en comparaison des impacts de ces perturbations sur les cours d'eau (Likens et al. 1970, Schindler et al. 1980, Bilby \& Bisson 1992, Beaty 1994, Hartman et al. 1996). Les quelques études récentes dans les lacs en Finlande et au Québec portent sur les impacts de la coupe forestière et/ou des feux sur la qualité des eaux (Rask et al. 1993, Lehmann 1994) ou les populations de salmonidés (Berub \& Levesque 1995). Aucune étude n'a prêté attention au zooplancton, une composante clé des réseaux pélagiques (Carpenter 1988, Carpenter \& Kitchell 1992). Une première recherche d'envergure sur la limnologie comparée des lacs de la forêt boréale a été entreprise en 1995 dans le cadre du Réseau de Centre d'Excellence en Gestion Durable de la Forêt (RCE-GDF). L'objectif primordial est de déterminer et comparer les impacts des feux naturels et des coupes forestières sur la qualité des eaux et les communautés aquatiques des lacs de la forêt boréale. L'objectif final est de développer des modèles de gestion prédisant les changements dans la qualité des eaux et le biota en fonction des variations dans la physiographie des bassins versants, la morphométrie des lacs et l'importance des perturbations par les feux ou les coupes de forêt.

Dans le cadre de cette étude préliminaire, nous décrivons et comparons la structure des communautés zooplanctoniques des lacs de référénce et des lacs ayant eu récemment un feu ou une coupe forestière d'importance sur leur bassin versant. La structure de la communauté zooplanctonique est caractérisée par différentes variables : 1) la composition taxonomique, la richesse spécifique et la densité du zooplancton, 2) la masse organique du limnoplancton (seston $>53 \mu \mathrm{m}$ ) et 3)'le biovolume du méso- et macrozooplancton ( $>250$ $\mu \mathrm{m})$. L'étude a pour objectif primordial de tester si ces indicateurs de la structure du zooplancton répondent aux perturbations des bassins versants par les feux ou les coupes à blanc et si la réponse du zooplancton varie selon le type de perturbations (feu vs.coupe).

\section{Matériel et méthodes}

\subsection{Sites d'étude}

L'étude a été réalisée en 1996 dans 38 lacs de l'écozone boréale du sud du Québec $\left(47-48^{\circ} \mathrm{N} ; 73-76^{\circ} \mathrm{W}\right)$. Dans ce secteur, la forêt mixte est composée de conifères et de feuillus (Picea mariana, Populus tremuloides, Betula papyrifera, Pinus divaricata) et subit chaque année des feux et des coupes de forêt sur de grandes étendues. En 1995, les compagnies forestières ont effectué des coupes à blanc sur près de 20000 ha avec $20 \mathrm{~m}$ de zone tampon autour des lacs et des cours d'eau. La même année, les feux de forêt ont ravagé près de 100000 ha sur le territoire d'étude (Gouvernement du Québec 1996, P. Darcy : com. pers.).

L'échantillonnage a été réalisé dans 20 lacs de référence aux bassins versants non perturbés, 9 lacs ayant subi en 1995 une coupe à blanc sur une partie de leur bassin versant et 9 lacs ayant eu en 1995 des feux de forêts sur la majorité de leur bassin versant. Les lacs non perturbés représentent le régime naturel de varia- 
tion des environnements lacustres en absence de perturbations majeures et constituent une référence pour comparer les impacts des perturbations naturelles (feux) et anthropiques (coupes). Les lacs de feux se conforment au modèle de perturbation naturelle avec une récurrence de feux importants sur un cycle de 50 à 200 ans selon les peuplements forestiers tandis que les lacs de coupes représentent le modèle de perturbation anthropique avec des cycles de coupes de 50 ans (Canadian Geographic 1996, Clark et al. 1996).

Le tableau 1 présente les caractéristiques morphométriques, chimiques, trophiques et ichtyologiques des lacs à l'étude ainsi que l'importance des perturbations sur le bassin versant pour chacun des groupes de lacs.

Tableau 1. Caractéristiques des trois groupes de lacs étudiés : moyenne saisonnière pour trois campagnes d'échantillonnage en juin, juillet et septembre 1996 (minimum, maximum). a . : biomasse de poissons capturés par filet et par nuit d'échantillonnage (BPUE) ; b: (pourcentage de la surface du bassin versant coupé ou brûlé).

Table 1. Characteristics of lakes studied in each group : summer mean for three sampling surveys in June, July and September 1996 (minimum, maximum). a. : Fish biomass per net and sampling night (BPUE) ; b. : (percentage of watershed area cut or burnt).

\begin{tabular}{cccc}
\hline GROUPES DE LACS & LACS DE & LACS DE COUPES & LACS DE FEUX \\
& REFERENCE & $(\mathrm{N}=9)$ & $(\mathrm{N}=9)$ \\
& $(\mathrm{N}=20)$ & & \\
\hline
\end{tabular}

\begin{tabular}{|c|c|c|c|}
\hline Aire du lac $\left(\mathrm{km}^{2}\right)$ & $0,46(0,16-0,86)$ & $0,59(0,20-2,38)$ & $0,43(0,19-0,66)$ \\
\hline Aire du bassin versant $\left(\mathrm{km}^{2}\right)$ & $2,68(0,60-5,85)$ & $4,13(0,93-12,94)$ & $5,71(0,78-22,2)$ \\
\hline Pente du bassin versant (\%) & $9,22(4,90-15,41)$ & $8,16(5,67-14,89)$ & $9,72(6,19-15,41)$ \\
\hline Profondeur maximale (m) & $12(7-22)$ & $14(4-25)$ & 17. $(10-37)$ \\
\hline Phosphore total $\left(\mu \mathrm{g} \cdot \mathrm{L}^{-1}\right)$ & $7,0(4,9-11,8)$ & $9,8(5,6-15,8)$ & $10,9(5,6-17,3)$ \\
\hline Azote total $\left(\mu \mathrm{g} \cdot \mathrm{L}^{-1}\right)$ & $251(181-399)$ & $303(205-450)$ & $370(207-859)$ \\
\hline Nitrate $\left(\mu \mathrm{g} \cdot \mathrm{L}^{-1}\right)$ & $6,5(<0,1-20,5)$ & $3,7(0,5-11,3)$ & $76,6(2,0-409,1)$ \\
\hline Carbone organique dissous (mg. $\mathrm{L}^{-1}$ ) & $5,1(2,8-9,2)$ & $8,0(3,0-13,3)$ & $5,6(3,1-8,0)$ \\
\hline Transparence (disque de Secchi, m) & $4,0(2,2-6,0)$ & $2,9(1,4-5,0)$ & $3,5(2,2-5,5)$ \\
\hline $\mathrm{pH}$ & $6,6(6,1-7,0)$ & $6,5(5,8-7,0)$ & $6,6(5,7-7,2)$ \\
\hline Chlorophylle $a\left(\mu \mathrm{g} \cdot \mathrm{L}^{-1}\right)$ & $1,9(1,1-3,1)$ & $2,4(1,5-3,2)$ & $3,1(1,8-4,1)$ \\
\hline Biomasse de cyprinidés BPUE (g) ${ }^{a}$ & $145(0-778)$ & $202(0-945)$ & $278(0-1374)$ \\
\hline Biomasse de prédateurs BPUE (g) ${ }^{\text {a }}$ & $2416(0-7804)$ & $2338(5-5012)$ & $3521(0-7949)$ \\
\hline Biomasse đe salmonidés BPUE $(\mathrm{g})^{\text {a }}$ & $824(0-6596)$ & $1088(0-3167)$ & $1702(0-7344)$ \\
\hline Biomasse de perchaudes BPUE $(\mathrm{g})^{a}$ & $185(0-1443)$ & $131(0-458)$ & $91(0-239)$ \\
\hline Biomasse de catostomes BPUE (g) ${ }^{a}$ & $1958(0-8213)$ & $1673(0-4978)$ & $2269(0-6529)$ \\
\hline Pourcentage de perturbation $(\%)^{\text {b }}$ & 0 & $44(7-96)$ & $90(50-100)$ \\
\hline
\end{tabular}


Les concentrations en éléments nutritifs (PT, $\mathrm{NT}, \mathrm{NO}_{3}$, COD), le $\mathrm{pH}$ et la biomasse du phytoplancton en chlorophylle $a$ ont été estimés dans la zone euphotique. La morphométrie des lacs et des bassins versants est assez similaire dans les trois groupes de lacs, exception faite d'un lac de coupes de plus grande surface $\left(12,94 \mathrm{~km}^{2}\right)$ et d'un lac de feux dont le bassin versant était très grand $\left(22,2 \mathrm{~km}^{2}\right)$. La qualité des eaux des lacs diffère entre les groupes de lacs et selon le type de perturbations (ANOVA; $\mathrm{P}<0.05$ ). Il y a davantage de phosphore total et d'azote total dans les lacs de feux et les lacs de coupes que dans les lacs de référence. Les nitrates sont en moyenne 10 à 20 fois plus concentrés dans les lacs de feux que dans les lacs de référence et les lacs de coupes tandis que les concentrations en carbone organique dissous sont plus élevées dans les lacs de coupes que dans les lacs de feux et les lacs de référence (R. Carignan, données non publiées). En conséquence, la transparence des eaux est plus fajble dans les lacs de coupes tandis que la biomasse du phytoplancton (Chl. a) est plus élevée dans les lacs de feux et les lacs de coupes que dans les lacs de référence.

Vingt-deux espèces de poissons ont été recensées dans les lacs à l'étude (Tableau 1), en particulier le meunier noir (Catostomus commersoni), des piscivores (Esox lucius, Lota lota, Stizostedion vitreum), des Salmonidés (Salvelinus fontinalis, Salvelinus namaycush, Coregonus clupeaformis), diverses espèces de cyprinidés (Semotilus corporalis, Coueisus plumbeus, Notemigonus crysoleucas, $N$. heterolepis, $N$. hudsonius, Phoxinus eos, $P$. neogaeus, Semotilus margarita, Notropis cornutus) et des perchaudes (Perca flavescens). La biomasse des différentes catégories ou espèces de poissons est très variable entre les lacs dans chacun des groupes.

L'importance des perturbations sur le bassin versant varie selon les groupes de lacs. Les lacs de coupes présentent un degré de perturbation moindre que les lacs de feux. Ainsi, parmi les lacs de coupes, la coupe de forêt s'est effectuée sur 33 à $96 \%$ du bassin versant sauf pour deux lacs où la coupe ne portait que sur 7 à $10 \%$ du bassin versant. Parmi les lacs de feux, les feux ont généralement ravagé plus de $90 \%$ de la surface du bassin versant, sauf dans un cas où seulement $50 \%$ du bassin versant a été brûlé.

\section{2. Échantillonnage et analyse du zooplancton}

Le zooplancton a été récolté en triplicat et à trois reprises durant l'été 1996 (juin, juillet, septembre) au site le plus profond de chaque lac. L'échantillonnage a été fait sur toute la colonne d'eau par halage vertical à l'aide d'un filet à contrelevier de $53 \mu \mathrm{m}$ d'ouverture de mailles (Filion et al. 1993). Chaque échantillon de zooplancton fut divisé en quatre fractions égales (1/4 du volume) à l'aide d'un séparateur Folsom. La première fraction a été réservée pour l'analyse taxonomique, la seconde pour l'évaluation de la masse organique du limnoplancton, la troisième pour l'analyse des biovolumes du méso- et macrozooplancton et la quatrième comme échantillon de référence.

L'analyse taxonomique a été faite sur les échantillons de juillet 1996 à partir de sous-échantillons de $10 \mathrm{ml}$ selon les méthodes développées par Pinel-Alloul \& Méthot (1984). La structure taxonomique de la communauté zooplanctonique de chacun des lacs a été établie en évaluant la richesse spécifique, les assemblages d'espèces, ainsi que la densité de chaque espèce et stades de développement (nauplies et copépodites) par unité de surface (ind $\mathrm{m}^{-2}$ ). La densité totale du zooplancton et des grands groupes taxonomiques (rotifères, cladocères, copépodes calanoïdes, copépodes cyclopoïdes) a été déterminée en sommant la densité des différentes espèces et stades de développement dans chaque groupe.

La biomasse du limnoplancton a été estimée en juin, juillet et septembre et pour l'ensemble de l'été 1996. Les estimés de biomasse ont été établis sur quatre classes de taille (53-100 $\mu \mathrm{m}, 100-200 \mu \mathrm{m}, 200-500$ $\mu \mathrm{m}$ et $>500 \mu \mathrm{m}$ ) par tamisage séquentiel du limnoplancton sur des filets Nitex (Salonen 1979, Martin 1993, Seda \& Dostalkova 1996). Les résidus de limnoplancton dans chacun des tamis ont été récoltés sur un filtre (Whatman GF/A), séchés à $40^{\circ} \mathrm{C}$ pendant 18 heures et pesés avec une microbalance analytique Cahn modèle $26( \pm 1 \mu \mathrm{g})$. Les filtres et résidus étaient ensuite incinérés à $500^{\circ} \mathrm{C}$ pendant 18 à 20 heures, puis pesés à nouveau. La différence entre les deux pesées correspond au poids sec sans cendre (PSSC) et représente la masse organique du limnnoplancton de chaque classe de taille. L'examen microscopique des différentes classes de taille de limnoplancton a montré que les classes de taille $<200 \mu \mathrm{m}$ étaient surtout composées de détritus organiques, de phytoplancton et de microzooplancton (rotifères, nauplies) tandis que les classes de taille $>200 \mu \mathrm{m}$ correspondaient au méso(petits cladocères et copépodites) et au macrozooplancton (daphnies, cyclopoïdes et calanoïdes adultes). La masse du limoplancton total par unité de surface sur l'ensemble de la colonne d'eau (mg.PSSC. $\mathrm{m}^{-2}$ ) correspond à la somme des PSSCs de chaque classe de taille.

L'analyse du biovolume du méso- et macrozooplancton (organismes $>250 \mu \mathrm{m}$ ) a été faite à l'aide d'un analyseur optique de particules (OPC-1L, Focal Technologies Inc., Darmouth, Canada) (Herman 1992, 
Sprules et al. 1992) à partir des échantillons de juillet 1996. L'échantillon de zooplancton est mis en suspension dans un aquarium, puis pompé à travers la cellule carrée de $21 \mathrm{~mm}$ d'arrête que traverse le faisceau infrarouge du compteur optique. Le passage de chaque particule à travers le faisceau infrarouge produit un signal électrique dont l'intensité est fonction de la surface projetée de la particule. Ce signal est ensuite converti en diamètre sphérique équivalent (DSE), soit le diamètre d'une sphère qui aurait produit le même signal. Les limites inférieure et supérieure de détection du compteur optique de particules sont de 267 et 14 $000 \mu \mathrm{m}$ (DSE), respectivement. Les données en DSE sont finalement converties en biovolumes $(\pi \times$ $\mathrm{DSE}^{3} / 6$ ) et le biovolume du zooplancton dans chaque classe de taille est établi en multipliant le nombre de particules incluses dans la classe de taille par le biovolume correspondant. Les spectres de taille sont constitués de onze classes volumétriques à progression logarithmique base 2 , soit de $2^{-7} \mathrm{~mm}^{3}$ (équivalent à 0,25 $\mathrm{mm}$ en diamètre sphérique équivalent, DSE) à $2^{3} \mathrm{~mm}^{3}$ (2,5 mm en DSE) (voir Fig. 5). Finalement, le biovolume total du zooplancton exprimé par unité de surface $\left(\mathrm{mm}^{3} \cdot \mathrm{m}^{-2}\right)$ sur l'ensemble de la colonne d'eau de la zone pélagique est égal à la somme des biovolumes de chaque classe de taille.

\subsection{Analyses statistiques}

Pour tester les effets des traitements (effets des perturbations : feux et coupes vs lacs de référence) sur le zooplancton des lacs de la forêt boréale, les valeurs moyennes (des trois réplicats) des variables du zooplancton dans chacun des lacs, ont été soumises à des ANOVAs à un critère de classification (i.e. groupes de lacs : référence, coupes, feux) et à des tests de Scheffe pour les comparaisons deux à deux des groupes de lacs (Sokal \& Rohlf 1981, SPSS Inc. 1990). De plus, une analyse de variance hiérarchique a permis d'évaluer comment se distribue la variance totale de la biomasse du limnoplancton et du biovolume du méso- et macrozooplancton entre les différentes sources de variation : inter-groupes ou inter-traitements $(n=3)$, inter-lacs $(n=38)$ et inter-réplicats $(n=3)$. Tous les tests ANOVA ont été effectués sur les données transformées $\left(\log _{10} \mathrm{x}\right)$, sauf pour les données de richesse spécifique (nombre d'espèces par lac) qui présentaient une distribution statistique normale. Finalement, afin d'évaluer les différences (inter-groupes, inter-lacs et inter-réplicats) dans les assemblages d'espèces, une analyse en coordonnées principales a été réalisée sur la matrice de similarité (coefficient de Steinhaus) des abondances des espèces de zooplancton ( $\mathrm{nb}$. d'individus par $\mathrm{m}^{2}$ ) en juillet 1996 dans les 38 lacs en incluant les trois répli- cats d'échantillonnage dans le cas de 9 lacs (3 lacs dans chacun des groupes) afin d'évaluer la variation intra-lac dans les assemblages d'espèces (Legendre \& Legendre 1984).

\section{Résultats}

\subsection{Composition et richesse spécifique du zoo- plancton}

En juillet 1996, le nombre total d'espèces de zooplancton dans l'ensemble des 38 lacs était en moyenne de 32 , dont 19 espèces de rotifères, 5 espèces de cladocères, 4 espèces de copépodes calanoïdes et 4 espèces de copépodes cyclopoïdes. On retrouvait en moyenne $29,2 \pm 1,2$ espèces de zooplancton dans les lacs de référence et légèrement plus dans les lacs de coupes $(31,2 \pm 2,4)$ et les lacs de feux $(34,6 \pm 1,5)$ (Fig. 1). Toutefois, cette légère augmentation de la richesse spécifique dans les lacs perturbés, comparativement aux lacs de référence, n'était pas statistiquement significative $(\mathrm{P}=0.09)$; elle était principalement attribuable à une faible hausse du nombre d'espèces de rotifères (17,5 dans les lacs de référence à 21,2 espèces dans les lacs de feux). Le nombre d'espèces de cladocères $(4,4$ à 5,3$)$, de copépodes calanoïdes $(3,0$ à 4,1$)$ et cyclopoïdes $(3,7$ à 4,0$)$ variait très peu entre les groupes de lacs. Les espèces les plus fréquentes et abondantes dans tous les lacs étaient Keratella cochlearis, Kellicottia longispina, Conochilus unicornis, Polyarthra remata, $P$. major, $P$. vulgaris, Conochiloides dossuarius, Gastropus stylifer, Filinia terminalis, Keratella crassa, $K$, taurocephala, Kellicottia bostoniensis, Trichocerca elongata, Ploesoma lenticulare et Polyarthra euryptera chez les rotifères, Bosmina spp., Daphnia longiremis, D. catawba, D. pulex, Diaphanosoma leuchtenbergianum et Holopedium gibberum chez les cladocères, Leptodiaptomus minutus et Epischura lacustris chez les copépodes calanoïdes, et Cyclops scutifer, Mesocyclops edax et Diacyclops bicuspidatus chez les copépodes cyclopoïdes. L'analyse en coordonnées principales sur les assemblages d'espèces en juillet 1996 dans les 38 lacs (Fig. 2) démontre que les lacs de référence, les lacs de coupes et les lacs de feux ne forment pas des groupes distincts mais se distribuent sur l'ensemble du premier plan d'ordination. Les deux premiers axes d'ordination expliquent seulement $23 \%$ de la variance totale dans les assemblages d'espèces et opposent des lacs de différents groupes (référence, coupe, feu) dont les communautés sont dominées par différentes associations d'espèces. Dans l'ensemble, les feux ou les coupes de forêt semblent donc avoir peu d'influence sur la richesse spécifique et la composition taxonomique du zooplancton des lacs de 


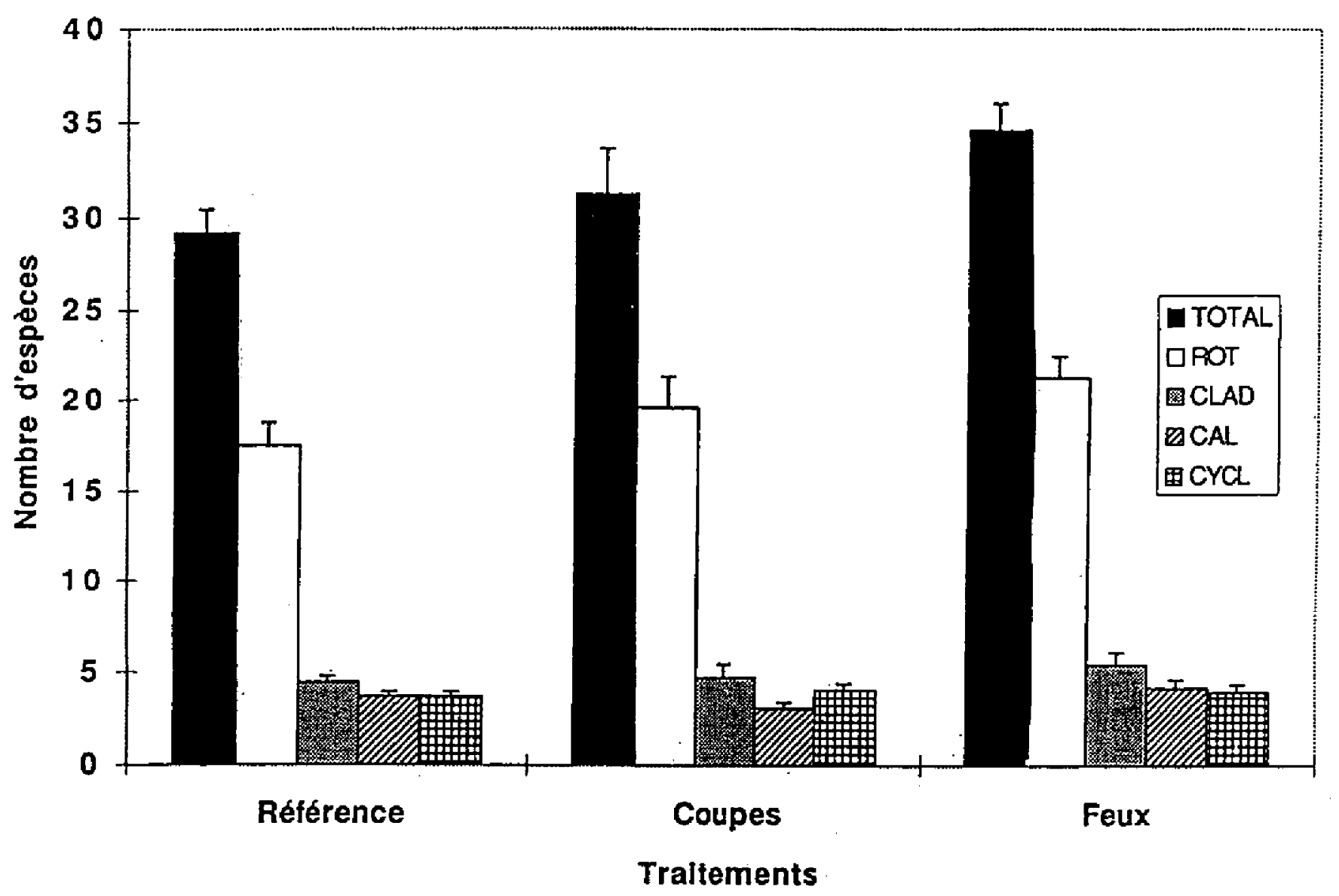

Fig. 1. Nombre (moyenne et erreur type) d'espèces de zooplancton (TOTAL), de rotifères (ROT), de cladocères (CLAD), de copépodes calanoïdes (CAL) et de copépodes cyclopoïdes (CYCL) dans les trois groupes de lacs (référence, coupes, feux) en juillet 1996.

Fig. 1. Species richness (mean and standard deviation) for zooplankton community (TOTAL), rotifers (ROT), cladocerans (CLAD), calanoid copepods (CAL), and cyclopoid copepods (CYCL) in the three groups of lakes (reference, cut, fire) in July 1996.

la forêt boréale. La variation intra-lac entre les réplicats de 9 lacs (points encerclés) est faible comparativement à la variation inter-lacs. Une ANOVA à un critère de classification (réplicat d'échantillonnage) effectuée pour comparer les coordonnées de chacun des trois réplicats des 9 lacs sur les deux premiers axes d'ordination démontre que la position des lacs ne varie pas d'un réplicat à l'autre $(\mathrm{n}=27, \mathrm{~F}=0.10, \mathrm{P}>0.99)$, ce qui indique que la variation intra-lac dans les assemblages d'espèces est négligeable.

\subsection{Abondance du zooplancton et des groupes taxo- nomiques}

En juillet 1996, l'abondance du zooplancton variait de $321.10^{3}$ à $4776.10^{3}$ ind. $\mathrm{m}^{-2}$ pour l'ensemble des 38 lacs avec $1060.10^{3}$ ind. $\mathrm{m}^{-2}$ en moyenne. Les rotifères étaient dominants (en moyenne : $704.10^{3}$ ind. $\mathrm{m}^{-2}$, $66 \%)$ suivis par les cyclopoïdes $\left(243.10^{3}\right.$ ind. $\mathrm{m}^{-2}$, $23 \%$ ), les calanoïdes $\left(89.10^{3}\right.$ ind. $\left.\mathrm{m}^{-2}, 8 \%\right)$ et les cladocères $\left(24.10^{3}\right.$ ind. $\left.\mathrm{m}^{-2}, 2 \%\right)$. L'abondance moyenne du zooplancton était semblable dans les lacs de référence $\left(851 \pm 93.10^{3}\right.$ ind. $\left.\mathrm{m}^{-2}\right)$ et les lacs de coupes ( 881 $\pm 161.10^{3}$ ind. $\left.\mathrm{m}^{-2}\right)$ mais augmentait par un facteur de 2 dans les lacs de feux $\left(1703 \pm 422.10^{3}\right.$ ind $\left.\mathrm{m}^{-2}\right)$ (ANOVA sur les données transformées $\left(\log _{10} \mathrm{x}\right): \mathrm{F}_{2,35}=$ 4,$74 ; \mathrm{P}=0.015$ ) (Fig. 3). Cette différence est surtout attribuable aux rotifères et aux copépodes cyclopoïdes.

\subsection{Biomasse du limnoplancton}

Pour l'ensemble de la saison estivale 1996 et à chacun des mois, la biomasse du limnoplancton était plus élevée dans les lacs de feux que dans les lacs de référence et les lacs de coupes (Fig.4). Pour l'ensemble des 38 lacs, la biomasse du limnoplancton total variait d'un minimum de $267 \mathrm{mg}$ PSSC. $\mathrm{m}^{-2}$ à un maximum de $1178 \mathrm{mg}$ PSSC. $\mathrm{m}^{-2}$ (poids sec sans cendres) et était en moyenne de $569 \pm 33 \mathrm{mg}$ PSSC. $\mathrm{m}^{-2}$. Au cours de l'été, la biomasse moyenne du limnoplancton total passait d'un maximum de $697 \mathrm{mg}$ PSSC. $\mathrm{m}^{-2}$ au mois de juin à $582 \mathrm{mg}$ PSSC. $\mathrm{m}^{-2}$ au mois de juillet et baissait à 


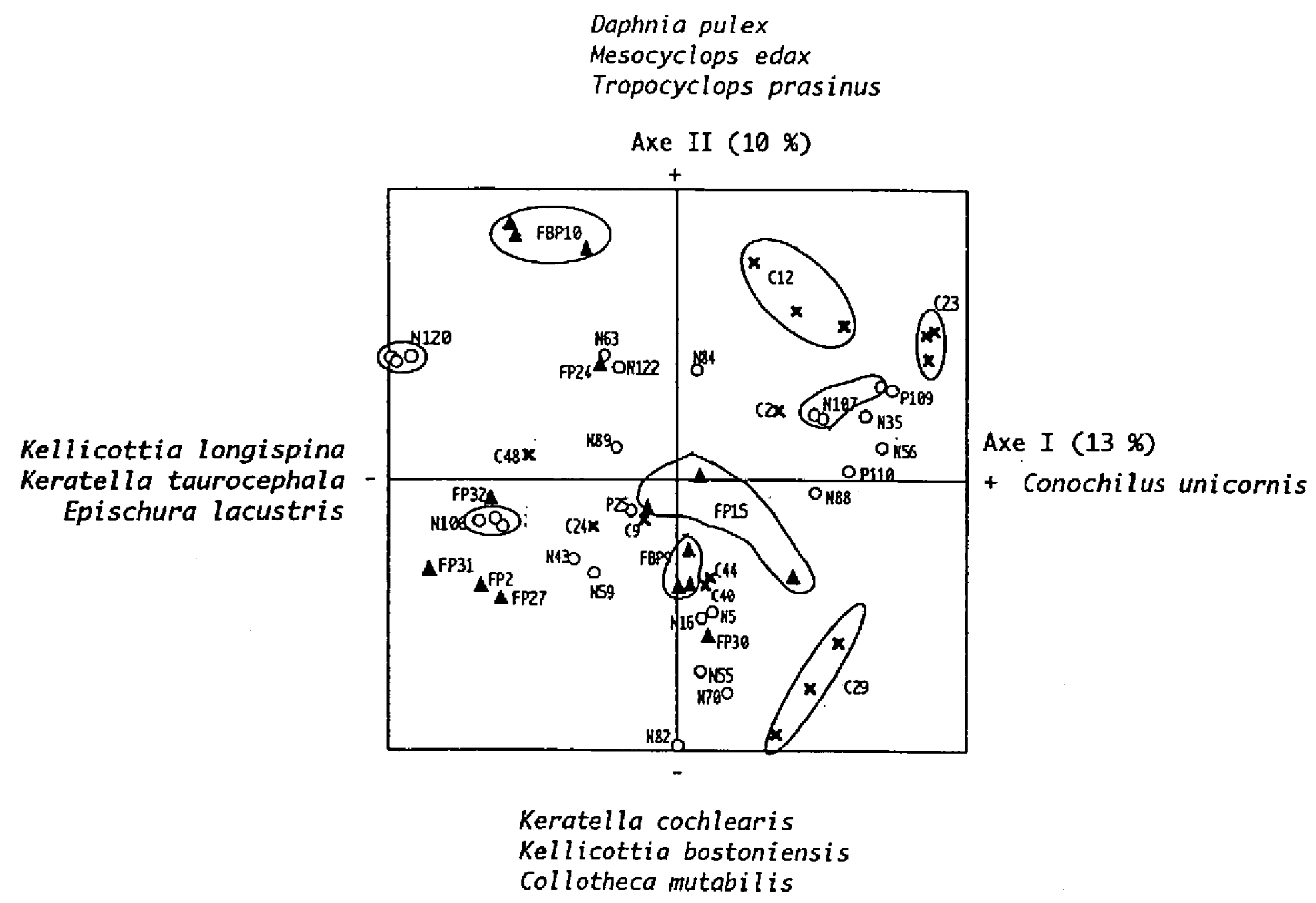

Fig. 2. Distribution đes lacs $(n=38$ ) et des réplicats d'échantillonnage encerclés (pour 9 lacs) dans le premier plan d'ordination de l'analyse en coordonnées principales basée sur la similarité des assemblages d'espèces de zooplancton dans les lacs de chacun des groupes (référence, coupes, feux) en juillet 1996 . N ou P : lacs de référence ; C : lacs de coupe; F : lacs de feux.

Fig. 2. Clustering of lakes $(n=38)$ and sampling replicates in circles (for 9 lakes) in the first PCA ordination plan based on the similarity of species assemblages in lakes in each group (reference, cut, fire) in July 1996. $\mathrm{N}$ or $\mathrm{P}:$ reference lakes ; C : cut lakes ; F : burnt lakes.

$427 \mathrm{mg}$ PSSC. $\mathrm{m}^{-2}$ au mois de septembre. L'analyse de variance réalisée sur les moyennes saisonnières $\left(\log _{10}\right.$ $\mathrm{x})$ a montré que la biomasse du limnoplancton total dans les lacs de feux $\left(800 \pm 76 \mathrm{mg}\right.$ PSSC. $\left.\mathrm{m}^{-2}\right)$ était significativement plus grande que celle observée dans les lacs de coupes $\left(477 \pm 56 \mathrm{mg}\right.$ PSSC. $\mathrm{m}^{-2}$ ) et les lacs de référence $\left(506 \pm 24 \mathrm{mg}\right.$ PSSC.m $\left.{ }^{-2}\right)\left(\mathrm{F}_{2,35}=10,79\right.$; $P=0.0002$ ). Il n'y avait pas de différences significatives dans la biomasse du limnoplancton total entre les lacs de coupes et les lacs de référence $(P=0.68)$. L'analyse de variance hiérarchique (groupes, lacs et réplicats) réalisée sur les données du mois de juillet 1996 a montré que $72 \%$ de la variance totale de la biomasse du limnoplancton était expliquée par les différences inter-lacs, $16 \%$ par les différences entre les groupes de lacs et $12 \%$ par les différences intra-lac entre les réplicats d'échantillonnage (Tableau 2).

\subsection{Biovolume du méso- et macrozooplancton}

La figure 5 présente les spectres de taille moyenne en biovolume du méso- et macrozooplancton pour chacun des groupes de lacs en juillet 1996. Pour l'ensemble des 38 lacs, le biovolume du zooplancton total (sommation des onze classes de taille) variait de 2288 à $66616 \cdot \mathrm{mm}^{3} \cdot \mathrm{m}^{-2}$ avec une moyenne de $12610 \pm 1$ $772 \mathrm{~mm}^{3} \cdot \mathrm{m}^{-2}$. Les lacs de feux étaient les plus riches avec $20972 \pm 6273 \mathrm{~mm}^{3} \cdot \mathrm{m}^{-2}$, suivis des lacs de coupes $\left(12577 \pm 2221 \mathrm{~mm}^{3} \cdot \mathrm{m}^{-2}\right)$ et des lacs de référence $\left(8862 \pm 912 \mathrm{~mm}^{3} \cdot \mathrm{m}^{-2}\right)$. L'augmentation des biovolumes de zooplancton dans les lacs de feux et les lacs de coupes était surtout attribuable à l'augmentation de la biomasse des classes de taille supérieures à $1,2 \mathrm{~mm}$ en DSÉ. Toutefois, l'analyse de variance à un critère de classification (inter-groupes) basée sur les données transformées $\left(\log _{10} x\right)$ du biovolume total du méso- et macrozooplancton indique que ces diffé- 


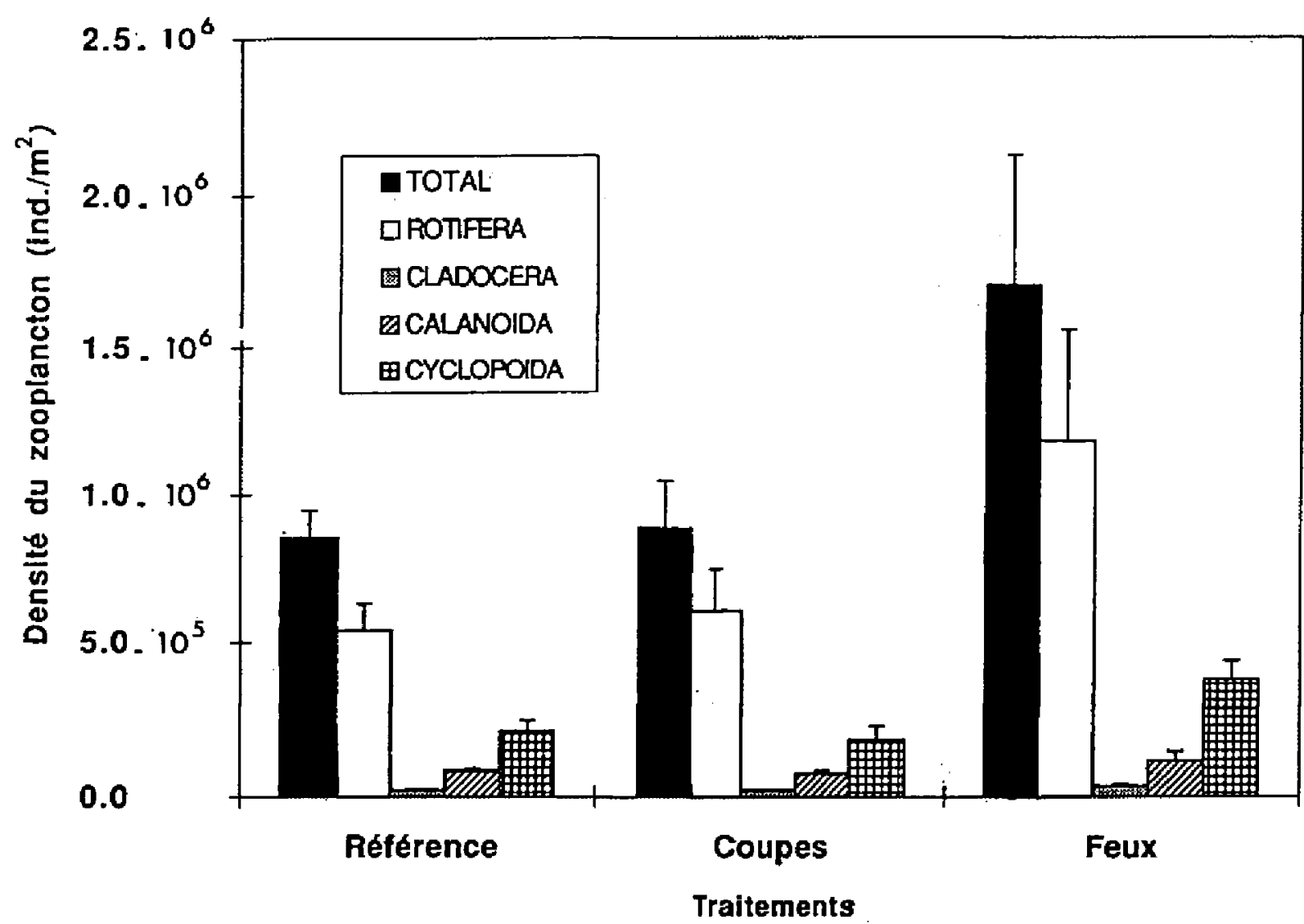

Fig. 3. Densité (moyenne et erreur type : ind. $\mathrm{m}^{-2}$ ) du zooplancton (TOTAL), des rotifères (ROTIFERA), des cladocères (CLADOCERA), des copépodes calanoïdes (CALANOIDA) et cyclopoïdes (CYCLOPOIDA) dans les trois groupes de lacs (référence, coupes, feux) en juillet 1996.

Fig. 3. Abundance (mean and error type : ind. $\mathrm{m}^{-2}$ ) of the zooplankton (TOTAL), rotifers (ROTIFERA), cladocerans (CLADOCERA), calanoid (CALANOIDA) and cyclopoid (CYCLOPOIDA) copepods in the three groups of lakes (reference, cut, fire) in July 1996.

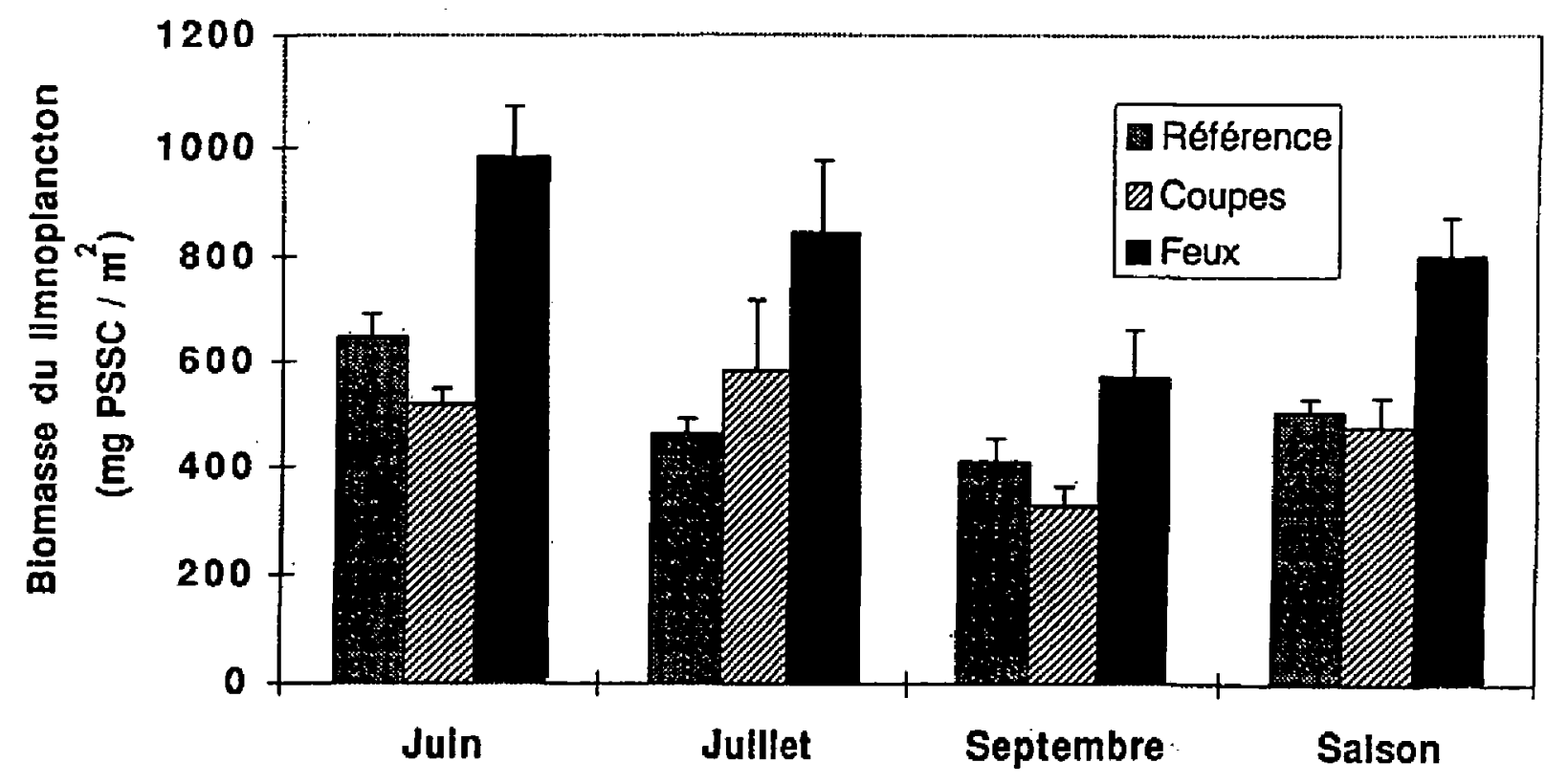

Fig. 4. Biomasse du limnoplancton (moyenne et erreur type : PSSC : $\mathrm{mg}^{-2} \mathrm{~m}^{-2}$ ) dans les trois groupes de lacs (référence, coupes, feux) en juin, juillet et septembre 1996 et pour l'ensemble de la saison estivale 1996.

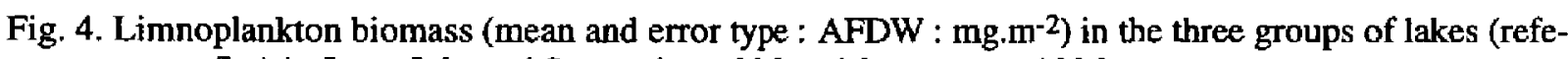
rence, cut, fire) in June, July and September 1996 and for summer 1996. 
Tableau 2. Partition de la variance totale de la biomasse du limnoplancton (A) et du biovolume du mésoet macrozooplancton (B) entre les différentes sources de variation (inter-groupes, inter-lacs et inter-réplicats). Données de juillet 1996.

Table 2. Partition of total variation in limnoplankton biomass (A) and méso-and macrozooplankton biovolume (B) among the different sources of variation (among groups, among lakes and among replicates). Data from July 1996.

\section{A: BIOMASSE DU LIMNOPLANCTON}

\begin{tabular}{llllllc}
\hline $\begin{array}{c}\text { Sources de } \\
\text { variation }\end{array}$ & $\%$ & $\begin{array}{c}\text { Nb. de degré } \\
\text { de liberté }\end{array}$ & $\begin{array}{c}\text { Variance } \\
\text { expliquée }\end{array}$ & $\begin{array}{c}\text { Valeur } \\
\text { de F }\end{array}$ & $\begin{array}{c}\mathrm{F} \\
\text { critique }\end{array}$ & $\begin{array}{c}\text { Seuil de } \\
\text { signification } \\
\text { (P) }\end{array}$ \\
\hline Groupes & 16 & 2 & 0,48 & 10,60 & 3,27 & 0.0001 \\
Lacs & 72 & 35 & & & & \\
Réplicats & 12 & 76 & & & & \\
\hline
\end{tabular}

B: BIOVOLUME DU MÉSO- ET MACROZOOPLANCTON

\begin{tabular}{|c|c|c|c|c|c|c|}
\hline $\begin{array}{c}\text { Sources de } \\
\text { variation }\end{array}$ & $\%$ & $\begin{array}{l}\text { Nb. de degré } \\
\text { de liberté }\end{array}$ & $\begin{array}{l}\text { Variance } \\
\text { expliquée }\end{array}$ & $\begin{array}{l}\text { Valeur } \\
\text { de F }\end{array}$ & $\underset{\text { critique }}{F}$ & $\begin{array}{c}\text { Seuil de } \\
\text { signification } \\
\text { (P) }\end{array}$ \\
\hline Groupes & 11 & 2 & 0,61 & 7,16 & 3,27 & 0.001 \\
\hline Lacs & 74 & 35 & & & & \\
\hline Réplicats & 15 & 76 & & & & \\
\hline
\end{tabular}
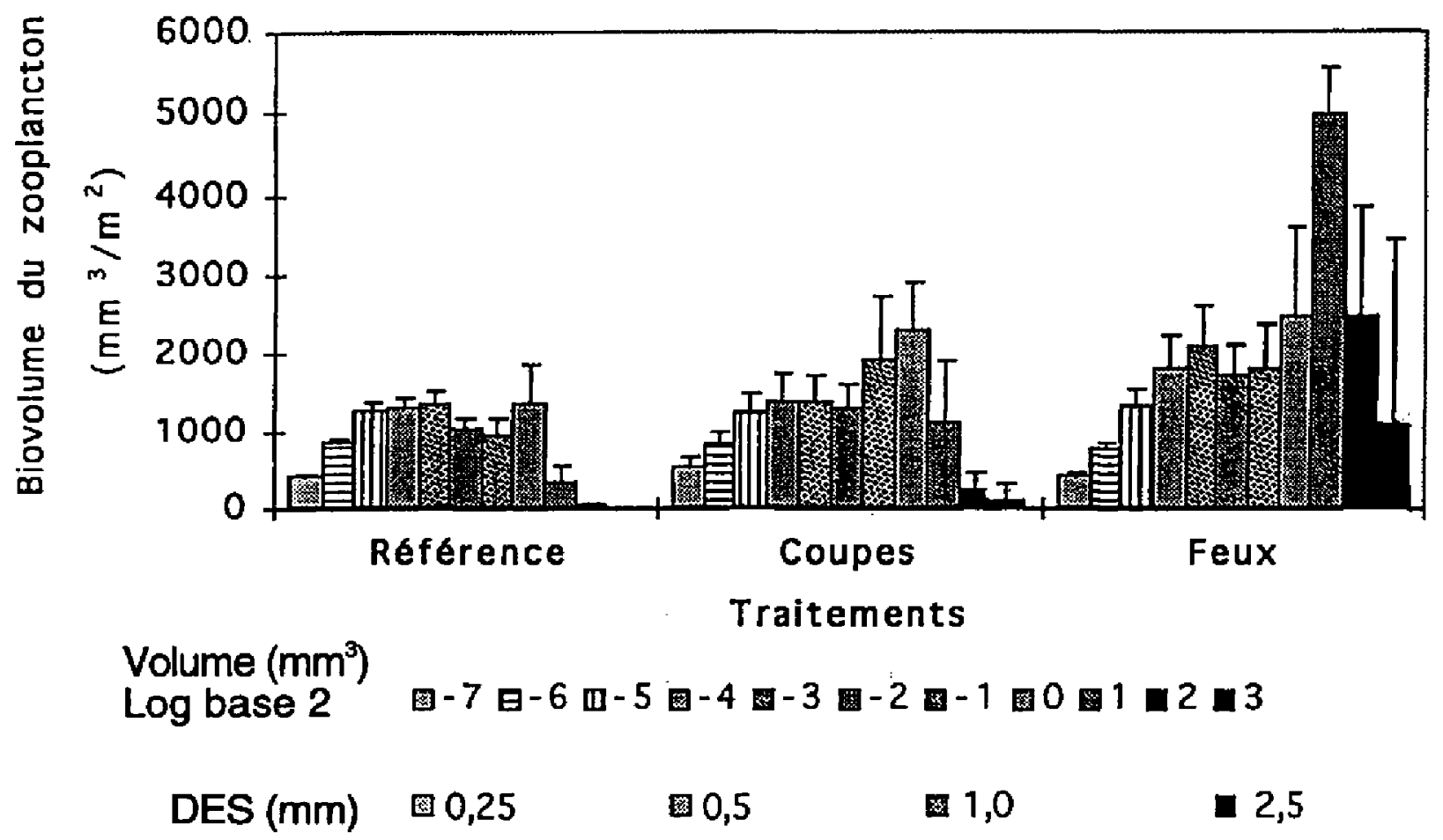

Fig. 5. Spectres de taille moyenne du méso- et macrozooplancton (en biovolume : $\mathrm{mm}^{-3} \cdot \mathrm{m}^{-2}$ ) pour chaque groupe de lacs (référence, coupes, feux) en juillet 1996.

Fig. 5. Mean size spectra of the meso- and macrozooplankton (in biovolume : $\mathrm{mm}^{-3} \cdot \mathrm{m}^{-2}$ ) for each group of lakes (reference, cut, fire) in July 1996. 
rences ne sont pas statistiquement significatives $\left(F_{2,35}\right.$ $=2,85 ; P=0,07)$. Ceci est du à une très forte variabilité inter-lacs dans chacun des groupes. En effet, l'analyse de variance hiérarchique (groupes, lacs et réplicats) a montré que $74 \%$ de la variance totale était attribuable aux variations inter- lacs, $11 \%$ aux variations entre les groupes et $15 \%$ aux variations intra-lac entre les réplicats (Tableau 2).

\section{Discussion}

\subsection{Effets des feux et des coupes de forêts}

Le zooplancton des lacs de la forêt boréale répond différemment aux perturbations du bassin versant causées par les feux et les coupes forestières. Bien que préliminaire, notre étude démontre clairement que les feux de forêts induisent une pousse trophique ascendante dans les écosystèmes planctoniques. Les lacs de feux contiennent environ 2 fois plus de zooplancton et de limnoplancton que les lacs de coupes et les lacs de référence. Cette poussée trophique serait attribuable aux apports importants de phosphore et surtout de nitrates après les feux et à l'augmentation subséquente de la biomasse du phytoplancton. Des effets comparables ont été notés dans les ruisseaux de la forêt boréale où les feux ont provoqué une hausse importante de toutes les formes d'azote et de phosphore (Schindler et al. 1980, Bayley et al. 1992, Beaty 1994) et une production accrue de périphyton et d'invertébrés benthiques (Vannote et al. 1980).

Par contre, la coupe forestière n'a pas d'effets ascendants sur la production de zooplancton dans les lacs de la forêt boréale, en dépit d'une légère augmentation des concentrations en phosphore et azote. L'augmentation du COD et de la couleur de l'eau après la coupe, en diminuant la transmission de la lumière, pourrait avoir indirectement limité le développement du phytoplancton et du zooplancton dans les lacs de coupes. L'augmentation de la biomasse du phytoplancton a d'ailleurs été moins forte dans les lacs de coupe que dans les lacs de feu. Dans les lacs de la forêt boréale du Canada et de Finlande, l'augmentation de la couleur de l'eau est associée à la hausse en COD et aux apports allochtones de matières humiques (Rasmussen et al. 1989, Kortelainen 1993). Un autre élément pouvant expliquer l'absence de réponses des communautés de zooplancton dans les lacs de coupe serait le plus faible niveau de perturbation. Ainsi, les coupes ont été faites sur 7 à $96 \%$ du bassin versant. À l'exception de quelques cas extrêmes (lacs C44 et C40 avec seulement 7,5 et $9,8 \%$ de leur bassin versant coupé et le lac C48 avec $96 \%$ de son bassin versant coupé), la majorité des lacs de coupes (6/9) ont eu 33 à $65 \%$ de l'aire du bassin versant déboisée. Une analyse comparée de la réponse du zooplancton dans les lacs de coupes indique en effet que les lacs les moins perturbés (C44 et C40) avaient les plus faibles niveaux de limnoplancton et de zooplancton tandis que le lac le plus perturbé (C48) se situait au deuxième rang dans les niveaux de limnoplancton et de zooplancton. Il semble donc que l'importance des coupes sur le bassin versant influence la réponse du zooplancton lacustre. Au niveau de la qualité des eaux, Lepistö et al. (1995) ont aussi noté l'absence de relation entre les apports en nitrates et l'importance des coupes forestières dans les lacs de la forêt boréale en Finlande et en Suède. Dans leur étude, l'importance des apports en nitrates variait beaucoup en fonction de la physiographie des bassins versants, des types de sols et du taux de repousse de la végétation ; seules, les coupes extrêmes sur la majorité du bassin versant ( $>90 \%$ de l'aire du bassin versant) avaient un effet significatif sur l'enrichissement trophique des lacs. Également, Lehmann (1994) n'a pas observé de relations significatives entre la surface des coupes forestières et les apports en nutriments (PT,NT, COD) dans les lacs de la Mauricie (Québec); la relation n'était significative que pour l'ensemble des lacs où les coupes avaient touché plus de $50 \%$ de la surface du bassin versant.

Notre étude ne démontre pas d'effet marqué des feux ou des coupes forestières sur la richesse spécifique et les assemblages d'espèces de zooplancton des lacs de la forêt boréale en dépit d'une légère augmentation du nombre d'espèces (en particulier des rotifères) dans les lacs perturbés comparativement aux lacs de référence. La richesse spécifique et les espèces dominantes du zooplancton des lacs à l'étude sont semblables à celles rapportées dans les lacs du Bouclier canadien du Québec et de l'Ontario (Pinel-Alloul et al. 1990, Keller \& Conlon 1994). En Nouvelle Angleterre, Stemberger \& Lazorchak (1994) ont par contre observé un effet des perturbations du littoral des lacs sur les assemblages de zooplancton. Le niveau de perturbation était relié au pourcentage du pourtour du lac utilisé pour l'agriculture et le développement urbain.

\subsection{Effets des environnements lacustres}

La plus grande variation des communautés de zooplancton d'un lac à l'autre, qu'en fonction des types de perturbations, souligne la difficulté de discerner les effets des perturbations anthropiques sur les composantes biotiques des écosystèmes aquatiques à cause de leur grande variabilité naturelle. Cela met aussi en évidence l'importance de faire des suivis à long terme (Schindler 1987) et de tenir compte de la variabilité naturelle entre les lacs et les bassins versants et en parti- 
culier des processus hydrologiques et chimiques à l'interface terre-eau pour mieux prédire les effets des perturbations (Lepistö et al. 1995, Allan \& Johnson 1997). En effet, plusieurs facteurs limnologiques peuvent avoir une influence sur la structure du zooplancton dans les lacs de la forêt boréale, en particulier la morphométrie des lacs et des bassins versants, le niveau trophique des lacs, la qualité chimique des eaux et les types de prédateurs (Confer et al. 1983, Keller \& Pitblado 1984, Magnan 1988, Dodson 1992, Keller \& Conlon 1994, Stemberger \& Lazorchak 1994, PinelAlloul et al. 1995). Ces facteurs peuvent avoir une influence indirecte sur les relations entre les communautés aquatiques et les perturbations du bassin versant.

Parmi les facteurs abiotiques, la profondeur et la surface des lacs peuvent influencer la structure du zooplancton en augmentant la richesse en espèces et l'abondance des espèces hypolimnétiques (Dodson 1992, Keller \& Conlon 1994). Les plus fortes densités de zooplancton et biomasses de limnoplancton observées dans les lacs de feux pourraient être en partie reliées au fait que ces lacs semblent en moyenne plus profonds que les lacs de coupe et les lacs de référence ( $17 \mathrm{~m}$ vs 14 et $12 \mathrm{~m}$ ). Cependant, cette différence n'est due qu'à un seul lac (FP27: $37 \mathrm{~m}$ ), les autres lacs étant de profondeurs comparables (10-20 m) aux lacs de coupes et de référence. Le niveau trophique des lacs et la qualité chimique des eaux, en particulier l'acidité et la couleur, ont aussi une très forte influence sur la diversité et la structure du zooplancton. On observe une baisse du nombre d'espèces de zooplancton dans les lacs à $\mathrm{pH}<5$ (Confer et al. 1983), et des associations d'espèces-types en fonction de l'acidification et de l'eutrophisation des lacs (Gannon \& Stemberger 1978, Bays \& Crisman 1983, Keller \& Pitblado 1984, PinelAlloul et al. 1990). Toutefois, ces facteurs devraient avoir eu peu d'influence dans notre étude où les valeurs de phosphore, d'azote et de $\mathrm{pH}$ étaient semblables et comparables à celles des lacs du Bouclier canadien (Papineau 1996).

Parmi les facteurs biologiques, l'augmentation de la biomasse du phytoplancton dans les lacs de feux est reliée à celle du zooplancton, indiquant que l'effet ascendant des apports en nutriments se transmet dans les maillons inférieurs (phyto- et zooplancton) des réseaux trophiques. Finalement, les fortes variations dans les communautés de poissons et dans l'abondance des poissons piscivores et planctivores entre les lacs dans chacun des groupes pourraient avoir exercé une forte influence dans le contrôle de la structure du zooplancton (Magnan 1988, Keller \& Conlon 1994, PinelAlloul et al. 1995). L'influence relative de ces facteurs abiotiques et biotiques sur la variation inter-lacs observée dans les variables du zooplancton sera analysée plus en détail ultérieurement à partir du suivi à longterme (1996-99).

\subsection{Réponses des différentes variables du zooplanc- ton}

La biomasse du limnoplancton, la densité du zooplancton et le biovolume du méso- et macrozooplancton ont sensiblement les mêmes réponses vis-à-vis des feux et des coupes forestières et peuvent donc servir d'indicateurs globaux des effets des perturbations environnementales d'ordre naturel ou anthropique. Par contre, la richesse spécifique et les assemblages d'espèces ne sont pas modifiés à court terme par les perturbations des bassins versants. Les divers types de variables utilisés dans le cadre de cette étude donnent une perspective différente sur la communauté zooplanctonique et offrent des applications variées comme indicateurs de changement dans les écosystèmes aquatiques. Ainsi, la biomasse du limnoplancton et le biovolume de zooplancton sont des indicateurs plus grossiers mais plus faciles à analyser que la composition taxonomique du zooplancton. Toutefois, ils ne sont pas spécifiques au zooplancton car les particules retenues sur les filets ou comptées par le compteur optique de particules peuvent être aussi bien des détritus, du phytoplancton que du zooplancton. Ces variables composites peuvent servir d'indicateurs globaux tandis que les variables taxonomiques permettront d'évaluer les réponses des différentes espèces aux gradients environnementaux et aux perturbations.

\section{Remerciements}

Cette étude est supportée par le Réseau de Centres d'Excellence en Gestion Durable de la Forêt (RCE-GDF) et financée par les programmes du CRSNG (Réseau de Centres d'Excellence, Subvention de recherche individuelle de B.P.A.) et de FCAR (centre et équipes de recherche du GRIL). Les biomasses de chlorophylle a ont été estimées par D. Planas (données non publiées) et les biomasses de poissons par P. Magnan (données non publiées). Nous les remercions pour leur collaboration.

Nous remercions Ginette Méthot, Claudette Blanchard pour leur participation à l'échantillonnage, Emma Mangas poúr l'analyse taxonomique du zooplancton et Isabelle St. Onge pour l'analyse du limnoplancton. L'organisation logistique et la réalisation des campagnes d'échantillonnage ont été assurées par Pierre Darcy et une équipe d'étudiants des cycles supérieurs (A. Patoine, E. Garcia) et du premier cycle (Claude Lefevre, Julie Trépanier, Ève BezaireDussault).

\section{Travaux citês}

Allan J.D. \& Johnson L.B. 1997. - Catchment-scale analysis of aquatic ecosystems. Freshwater Biol., 37 : 107-111. 
Bayley S.E., Schindler D.W., Beaty K.G., Parker B.R. \& Stainton M.P. 1992. - Effects of multiple fires on nutrients yields from streams draining boreal forest and fen watersheds : nitrogen and phosphorus. Can. J. Fish. Aquat. Sci., 49 : 584-596.

Bays J.S. \& Crisman T.L. 1983. - Zooplankton and trophic state relationships in Florida lakes. Can. J. Fish. Aquat. Sci., 40 : 18131819.

Beaty K.G. 1994. — Sediment transport in a small stream following two successive forest fires. Can. J. Fish. Aquat. Sci., $51: 2723-$ 2733.

Berub P. \& Lévesque F. 1995. - Analyse des données d'exploitation de l'omble de fontaine en relation avec les interventions forestières dans la réserve faunique Mastigouche. Gouvernement du Québec, Ministère de l'Environnement et de la Faune. 25 p.-

Bilby R.E. \& Bisson P.A. 1992, - Allochtonous versus autochtonous organic matter contributions to the trophic support of fish populations in clear-cut and old-growth forested streams. Can J. Fish. Aquat. Sci., 49 : 540-551.

Canadian Geographic. 1996. - L'atlas national du Canada. La forêt boréale : carte-affiche. Ressources naturelles Canada. Service canadien des forêts. Gomatique Canada.

Carpenter S. R. 1988.(Ed.). - Complex interactions in lake communities. Springer-Verlag, New York : $283 \mathrm{p}$.

Carpentér S.R. \& KitcheII J.F. 1992. - Trophic cascade and biomanipulation : Interface of research and management. A reply to the comment by DeMelo et al.. Limnol. Oceanogr., 37 : 208-213.

Clark J.S., Royall P.D. \& Chumbley C. 1996. - The role of fire during climate change in an eastern deciduous forest at Devil's Bathub, New York. Ecology 77 : 2148-2166.

Confer J.L., Kaaret T. \& Likens G.E. 1983. — Zooplankton diversity and biomass in recently acidified lakes. Can. J. Fish. Aquat. Sci., $40: 36-42$.

Dodson S.I. 1992: - Predicting crustacean zooplankton species richness. Limnol. Oceanogr. $37: 848-856$.

Filion J-M., Chain P. \& Futter M. 1993. - Cantilevering vertical tow nets to reduce tow-line induced zooplancton avoidance. $J$. Plankton Res., 15 (5) : 581-587.

Forestry Canada. 1992. - Compendium of Canadian Forestry Statistics. National Forestry Database Program. Forestry Canada. Ottawa. Ontario : $650 \mathrm{p}$.

Gannon J.E. \& Stemberger R.S. 1978. - Zooplankton (especially Crustaceans and Rotifers) as indicators of water qualitey. Trans. Amer. Micros. Soc., 9 : 16-35.

Gouvernement du Québec. 1996. - Rapport sur l'état des forêts québécoises 1990-1994. À l'heure du développement durable. Une foresterie en constante évolution. Ministère d'état des Ressources naturelles. $163 \mathrm{p}$.

Hansson L. 1992. - Landscape ecology of boreal forest. Trends in Ecology and Evolution, $7: 9-15$.

Hartman G.F., Scrivener J.C. \& Miles M.J. 1996. - Impact of logging in Carnation Creek, a high-energy coastal stream in Bristish Columbia, and their implication for restoring fish habitat. Can.J. Fish. Aquat. Sci., 53 (Suppl. 1) : 237-251.

Herman A.W. 1992. - Design and calibration of a new optical plankton counter capable of sizing small zooplankton. Deep Sea Res. 39A : 395-415.

Johnson E.A. 1992. - Fire and Vegetation Dynamics : Studies From North American Boreal Forest. U.K. Cambridge University. 65 p.

Keller W. \& M. Conlon. 1994. - Crustacean zooplankton communities and lake morphometry in Precambrian Shield Lakes. Can. J. Fish. Aquat. Sci., 51 : 2424-2434.

Keller W. \& Pitblado J.R. 1984. - Crustacean plankton in northeastern Ontario lakes subjected to acidic deposition. Water, Air, Soil Pollution, 23 : 271-291.

Kortelainen P. 1993. - Content of total organic carbon in Finnish lakes and its relationship to catchment characterisitcs. Can. J. Fish. Aquat. Sci., $50: 1477-1483$.
Legendre L. \& Legendre P. 1984. - Ecologie numérique. - Tome 2. La structure des données écologiques. Masson et Presses de l'Université du Québec (Ed.). 335 p.

Lepistö A., Andersson L. Arheimer B. \& Sundblad K. 1995. - Influence of catchment characteristics, forestry activities and deposition on nitrogen export from small forest catchments. Water, Air and Soil Pollution, 84 : 81-102.

Likens G.E., Bormann F.H., Johnson N.M., Fisher D.W. \& Pierce R.S. 1970. - Effects of forest cutting and herbicide treatment on nutrient budgets in the Hubbard Brook Watershed Ecossytem. Ecological Monographs, $40: 23-47$.

Lehmann R. 1994. - Forest clearance and lake water qualitéy in the Canadian Shield. Master Thesis. Univ. McGill, Canada, 73 p.

Magnan P. 1988. - Interactions between brook charr, Salvelinus fontinalis and non-salmonid species: ecological shift, morphological shift, and their impact on zooplankton communities. Can. J. Fish. Aquat. Sci., 45 : 999-1009.

Martin C. 1993. - Analyse comparative du seston estival de 9 lacs du Jura français. Annls. Limnol., $29: 365-381$.

Papineau M. 1996. - Small lake water chemistry in Southem Québec. Water Qual. Res. J. Canada., 31 : 163-174.

Pinel-Alloul B. \& Méthot G. 1984. - Analyse multidimensionnelle de l'évolution du zooplancton durant la mise en eau de trois réservoirs du Nord du Québec, Canada. Verh. Intemat. Verein. Limnol., 22 : 1444-1455.

Pinel-Alloul B., Méthot G., Verreault G. \& Vigneault Y. 1990. Zooplankton species association in Quebec lakes : variation with abiotic factors, including natural and anthropogenic acidification. Can. J. Fish. Aquat Sci., 47: 110-121.

Pinel-Alloul B., Niyonsenga T. \& Legendre P. 1995. - Spatial and environmental components of freshwater zooplankton structure. Ecoscience, 2 : 1-19.

Rask M., Arvola L. \& Salonen S. 1993. - Effects of catchment deforestation and burning on the limnology of a small forest lake in Southern Finland. Verh. Internat. Verein. Limnol., 25 : 525-528.

Rasmussen J.B., Godbout L. \& Schallenberg B. 1989. - The humic content of lake water and its relationships to watershed and lake morphometry. Limnol. Oceanogr., 34 : 1336-1343.

Salonen K. 1979. - A versatile method for the rapid and accurate deternination of carbon by high temperature combustion. Limnol. Oceanogr., $24: 177-183$.

Schindler D.W. 1987. - Detecting ecosystem responses to anthropogenic stress. Can. J. Fish. Aquat. Sci., (Suppl. 1) : 6-25.

Schindler D.W., Newbury R.W., Beaty K.G., Prokopowich J., Ruszczynski T. \& Dalton J.A. 1980. - Effects of windstrom and forest fire on chemical losses from forested watersheds on the qualitéy of receiving streams. Can. J. Fish. Aquat. Sci.; 37 : 328-334.

Seda J. \& Dostalkova I. 1996. - Live sieving of freshwater zooplankton: a technique for monitoring community size structure. $J$. Plankton Res., 18 : 513-520.

Sokal R.R. \& Roff F.J. 1981. - Biometry : the principles and practice of statistics in biological research. W.H. Freeman \& Co., New York. : $413 \mathrm{p}$.

Sprules W. G., Bergstrom B., Cyr H., Hargreaves B.R., Kilham S.S., Matsushita K., Stemberger R. \& Williams R.1992. - Non-video optical instruments for studying zooplankton distribution and abundance. Arch. Hydrobiol. Beih. Ergebn. Limnol. 36 : 45-58.

SPSS Inc. 1990. - SPSS/PC + manuals : advanced statistics, tables, statistics 4.0 Base manual, and categories. Chicago, II.

Statistics Canada. 1994. - Human Activity in the Environment. 1994. Ministry of Industry, Science and Technology Catalogue II509E June 1994.

Stemberger R.S. \& Lazorchak J.M. 1994. - Zooplankton assemblage responses to disturbance gradients. Can. J. Fish. Aquat. Sci., $51: 2435-2447$.

Vannote R.L., Minshall G.W., Cummins K.W., Sedell J.R. \& Cushing C.E. 1980. - The river continuum concept. Can. J. Fish. Aquat. Sci., 37 : 370-377. 